YA.B. BAZALIY

University of South Carolina

(712 Main St. Columbia SC 29208,USA; e-mail: bazaliy@mailbox.sc.edu)

\title{
INTERPRETATION OF THE SPIN TORQUE SIGN CHANGE IN F/N/F STRUCTURES IN TERMS OF A MECHANICAL ANALOGY
}

\begin{abstract}
A useful interpretation of the spin-accumulation and spin-current distributions in magnetic nanostructures with diffusive transfer has been discussed. A mathematically equivalent mechanical system was proposed that provides an intuitive understanding of the dependence of the spin-transfer torque values on various parameters. In particular, it gives a clear explanation for the sign change of the spin-transfer torque in asymmetric $F / N / F$ structures.

Ke ywords: spin transfer, diffusion mode, spintronics.
\end{abstract}

\section{Introduction}

The electric current flow through magnetic structures with spatially inhomogeneous magnetization $\mathbf{M}(\mathbf{r})$ gives rise to the emergence of torques that are proportional to the current. Those torques are associated with the spin-transfer process and are called spintransfer torques [1-4]. The spin-transfer phenomenon is based on the exchange interaction $J(\mathbf{s} \cdot \mathbf{M})$ between the spins $\mathbf{s}$ of moving electrons and the ferromagnet magnetization $\mathbf{M}$. It results in the appearance of the torque $\mathbf{T}=J[\mathbf{M} \times \mathbf{s}]$ acting on $\mathbf{M}$.

Let us imagine a nonuniformly magnetized ferromagnet. In the equilibrium state, the spin density of moving electrons, $\langle\mathbf{s}\rangle$, is directed along the vector $\mathbf{M}(\mathbf{r})$ at every point $\mathbf{r}$, so that $\mathbf{T}=0$. The situation changes, if an electric current is present. The current brings electrons to the point $\mathbf{r}$ from distant regions, where their spins were oriented at a certain angle with respect to $\mathbf{M}(\mathbf{r})$. As a result, there emerges a nonequilibrium state with nonparallel vectors $\langle\mathbf{s}\rangle$ and $\mathbf{M}$, and, accordingly, a nonzero torque $\mathbf{T}$. The imbalance degree and, hence, the torque value are proportional to the current strength.

This physical picture can also be described in terms of an additional angular momentum $\hbar / 2$ transported by the spin of every moving electron [1-6]. In the framework of this approach, the torque is associated with the parameters of the spin currents $\mathbf{j}_{s}$. Let us

(C) YA.B. BAZALIY, 2020

ISSN 2071-0194. Ukr. J. Phys. 2020. Vol. 65, No. 10 consider a physically infinitesimal element in a ferromagnet. The spin density of moving electrons in this element, $\langle\mathbf{s}\rangle$, can change for two causes: (i) the exchange interaction generates a torque $\mathbf{T}_{s}$, which acts on $\langle\mathbf{s}\rangle$; and (ii) the input and output spin currents do not compensate each other, so that $\langle\mathbf{s}\rangle$ changes owing to the electron transfer process. In the stationary state, $\langle\mathbf{s}\rangle=$ const, and $\mathbf{T}_{s}$ must be exactly compensated by the imbalance from item (ii). In a continuous medium, such an imbalance is equal to the divergence of the spin current. Furthermore, since the existence of the torques $\mathbf{T}$ and $\mathbf{T}_{s}$ is a result of the same exchange interaction, one has $\mathbf{T}=-\mathbf{T}_{s}$ (action and reaction are equal and opposite). As a result, the spin-transfer torque $\mathbf{T}(\mathbf{r})$ in the stationary state is determined as the spin-current divergence at the point $\mathbf{r}$.

This work deals with nanostructures consisting of the layers of uniformly magnetized ferromagnets $(\mathrm{F})$ and nonmagnetic normal metals (N). In such structures, the spatial change of $\mathbf{M}$ occurs in the form of jumps at the interfaces between the layers of different materials. Experimentally, the spin transfer is often observed in nano-wires about $100 \mathrm{~nm}$ in diameter made of a normal metal and containing two ferromagnetic layers (Fig. 1).

If the magnetization directions in the F-layers are described by the unit vectors $\mathbf{m}_{1}$ and $\mathbf{m}_{2}$, then the calculations bring about the following formulas for the torques applied to the magnetizations of the first 


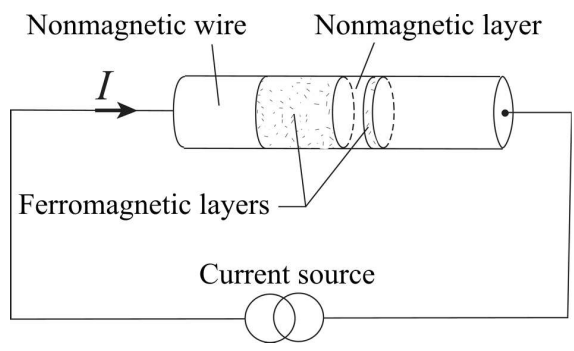

Fig. 1. Schematic diagram of a spin-transfer device

and second layers $[4,7,8]$ :

$$
\begin{aligned}
& \mathbf{T}_{1}=-\frac{I \hbar}{2 e} g_{1}(\theta)\left[\mathbf{m}_{1} \times\left[\mathbf{m}_{2} \times \mathbf{m}_{1}\right]\right] \\
& \mathbf{T}_{2}=\frac{I \hbar}{2 e} g_{2}(\theta)\left[\mathbf{m}_{2} \times\left[\mathbf{m}_{1} \times \mathbf{m}_{2}\right]\right] .
\end{aligned}
$$

Here, $I$ is the electric current in the wire, $e<0$ the electron charge (so that $I / e=j_{0}$ is the particle flux), $\theta$ the angle between the vectors $\mathbf{m}_{1}$ and $\mathbf{m}_{2}$, and $g_{1,2}(\theta)$ are the spin-transfer "efficiency functions". The minus sign in the first formula reflects the symmetry of the effect, namely, the fact that a ferromagnet located upstream along the positive current can be regarded as being located downstream along the negative current. Although the spintransfer torques arise owing to the interaction of ferromagnets through the motion of electrons between them, the sum $\mathbf{T}_{1}+\mathbf{T}_{2} \neq 0$. The total torque of the magnetic layers does not have to vanish, because the layers form an open system and can exchange the torque with other wire sections.

The efficiency functions depend on the electron transport mode, the material parameters, and the device geometry. Their dependence on the angle $\theta$ has been considered in many publications. In the first studies, these functions were taken to be positive. The following arguments were presented in favor of this assumption. When electrons from the first ferromagnetic layer $\mathrm{F}_{1}$ fly into the normal slab, they are spin-polarized along the vector $\mathbf{m}_{1}$. Afterward, when they reach the layer $\mathrm{F}_{2}$, their spins have to rotate by the angle $\theta$ (on average) with respect to the direction of the vector $\mathbf{m}_{2}$. The necessity for the spins to rotate makes it difficult for electrons to cross the interface and thus increases the interface resistance. The other flip of this effect is that the current from an external source must induce torques that would change the orientation of the magnetizations in the direc- tion corresponding to the resistance reduction, i.e., it would bring $\mathbf{m}_{2}$ closer to $\mathbf{m}_{1}{ }^{1}$. The $\mathbf{T}_{2}$ torque direction agrees with such a consideration provided the condition $g_{2}>0$ holds.

However, the later calculations [9-11] showed that an unexpected deviation from this conclusion arises even in the framework of the diffusion model: for asymmetric structures with the layers $\mathrm{F}_{1}$ and $\mathrm{F}_{2}$ made from different materials, the function $g(\theta)$ can change the sign. This result is used in various projects dealing with the generation of magnetization precession by means of a direct current $[10,12,13]$. The calculations in the diffusion regime are simple ideologically, but often produce long and cumbersome algebraic expressions. In this connection, there arises the need for the understanding of the alternating-sign character of $g(\theta)$ at the qualitative level. In this work, we demonstrate how the alternating-sign behavior of the efficiency function can be intuitively interpreted with the help of a mechanical analogy and how such an approach allows the sign of the efficiency function to be determined without carrying out detailed calculations.

\section{Diffusion-Transfer Model}

\subsection{Bulk equations}

In structures where the electron mean free path is much shorter than the thicknesses of the layers (see Fig. 1), the transport processes have a diffusive character. Furthermore, if the electric current is uniform over the nano-wire cross-section, then all physical parameters will depend only on the $x$-coordinate reckoned along the wire. The ferromagnetic layers are described by two diffusion coefficients, $D_{\uparrow}$ and $D_{\downarrow}$, for electrons with spins oriented in parallel (spin-up) and antiparallel (spin-down) to the vector $\mathbf{m}$. The normal layer is described by a single diffusion coefficient $D_{\mathrm{N}}$ common for all electrons. In an infinite ferromagnetic wire, the electric current from an external source generates the spin current $\mathbf{j}_{s}=p j_{0} \mathbf{m}$, where $p=\left(D_{\uparrow}-D_{\downarrow}\right) /\left(D_{\uparrow}+D_{\downarrow}\right)$ is the spin polarization of the current, and $j_{0}$ is the particle flux. If the prob-

1 The logic of this conclusion reminds the logic used while considering a rubber tube through which water is pumped under pressure. Since we know that it would be easier for water to pass through a tube with a larger diameter, we may conclude that the pumping has to stretch the tube diameter. In other words, we come to the Le Chatelier-Brown principle. 
lem is one-dimensional, all currents flow along the $x$-axis. Therefore, $j_{0}$ is a scalar and $\mathbf{j}_{s}$ a vector in the spin space.

At the interfaces between the layers, the electric current also induces a non-equiulibrium accumulation of the spin, $\delta \mathbf{s}[14,15]$, with $\delta \mathbf{s}=\langle\mathbf{s}\rangle$ in a normal metal and $\delta \mathbf{s}=\langle\mathbf{s}\rangle-\mathbf{s}_{\text {eq }}$ in a ferromagnet, where $\mathbf{s}_{\mathrm{eq}}$ is the nonzero spin density at equilibrium. Let us consider the case of strong ferromagnets, where the splitting $J$ of the energy bands of the spin-up and spin-down electrons has the same order of magnitude as the Fermi energy $\varepsilon_{\mathrm{F}}$. In this case, the vectors $\mathbf{s}_{\mathrm{eq}}$ and $\delta \mathbf{s}$ in the bulk of ferromagnets are always oriented in parallel to the vector $\mathbf{m}[8,16,17]$.

If a spin current with an arbitrary polarization falls on the $\mathrm{N} / \mathrm{F}$ interface from the normal-metal side, then the relaxation of its polarization direction to $\mathbf{m}$ occurs in a near-interface zone with the thickness $\lambda_{J} \sim \hbar v_{\mathrm{F}} / J$. In the case $J \sim \varepsilon_{\mathrm{F}}$, this thickness is narrower than the electron mean free path length. As a result, in the diffusion approximation, the relaxation of the spin current component perpendicular to $\mathbf{m}$ is described by the boundary conditions rather than bulk equations. It is important to emphasize that the condition of a strong ferromagnet, i.e. $J \sim \varepsilon_{\mathrm{F}}$, and the resulting rapid ordering of the spins in the $\mathbf{m}$ direction do not require the spin current to be almost completely polarized, $p \rightarrow 1$. The current polarization is determined not only by the band splitting $J$, but also by the velocities and the scattering parameters of spin-up and spin-down electrons. Therefore, it can be low even in strong ferromagnets. Vice versa, the limit $p \rightarrow 0$ does not imply a transition from ferromagnet to normal metal.

It is known $[9,18,19]$ that the diffusive accumulation of the spin in ferromagnets and normal metals can be characterized by the splitting of chemical potentials of electrons with spins up and down with respect to the direction $\delta \mathbf{s}, \mu_{s}=\mu_{\uparrow}-\mu_{\downarrow}$. It is convenient to describe this splitting using the vector $\boldsymbol{\mu}_{s}=\mu_{s} \mathbf{m}$, where the unit vector $\mathbf{m}$ coincides with $\mathbf{m}_{1,2}$ in the ferromagnets and is directed along $\delta \mathbf{s}$ in the normal metal. In the diffusion approximation, the particle flux and the spin current are determined by the gradients of $\boldsymbol{\mu}_{s}$ and the electrochemical potential $\mu_{0}$ as follows: in a ferromagnet,

$j_{0}=-\left(D_{\uparrow}+D_{\downarrow}\right)\left[\nabla_{x} \mu_{0}+p \frac{\nabla_{x} \mu_{s}}{2}\right]$,

ISSN 2071-0194. Ukr. J. Phys. 2020. Vol. 65, No. 10
$\mathbf{j}_{s}=-\left(D_{\uparrow}+D_{\downarrow}\right)\left[p \nabla_{x} \mu_{0}+\frac{\nabla_{x} \mu_{s}}{2}\right] \mathbf{m}$,

and, in a normal metal,

$j_{0}=-D_{\mathrm{N}} \nabla_{x} \mu_{0}$,

$\mathbf{j}_{s}=-D_{\mathrm{N}} \frac{\nabla_{x} \boldsymbol{\mu}_{s}}{2}$.

Since the current $j_{0}$ is constant at any point of the one-dimensional structure, we can rewrite the expression for the spin current in a ferromagnet in the form

$\mathbf{j}_{s}=\left(p j_{0}-D_{\mathrm{F}} \frac{\nabla_{x} \mu_{s}}{2}\right) \mathbf{m}$,

where $D_{\mathrm{F}}=4 D_{\uparrow} D_{\downarrow} /\left(D_{\uparrow}+D_{\downarrow}\right)$.

The distribution of spin accumulations in each layer is determined by the transport-relaxation equation -

$\frac{d \boldsymbol{\mu}_{s}}{d t}+\frac{1}{\rho} \operatorname{div} \mathbf{j}_{s}=-\frac{\boldsymbol{\mu}_{s}}{\tau}$

where $\tau$ is the spin relaxation time for the given material, $\rho=d s / d \mu_{s}$ is a coefficient associated with the densities of states for two spin directions, and $\mathbf{j}_{s}$ is defined by formulas (3) and (4). In the onedimensional stationary case (to within we restrict ourselves below),

$\frac{1}{\rho} \frac{d \mathbf{j}_{s}}{d x}=-\frac{\boldsymbol{\mu}_{s}}{\tau}$.

Hence, the problem of finding the spin currents becomes completely separated from the problem concerning the electric potential distribution. The former is reduced to the solution of Eq. (5) with the corresponding boundary conditions at the interfaces between the layers.

\subsection{Boundary Conditions}

If an electric current runs through a multilayer structure, the behavior of the potential $\mu_{0}$ at the interfaces depends on the ratio between the interface resistance and the resistivity of the material in each layer. In particular, if the interface resistance is low, the approximation of a continuous electric potential across the interface can be used. In this approximation, the current passing through a "transparent" interface does not induce a $\mu_{0}$-jump across it.

At first glance, the case of spin transfer seems to be quite different. Since the perpendicular component of 


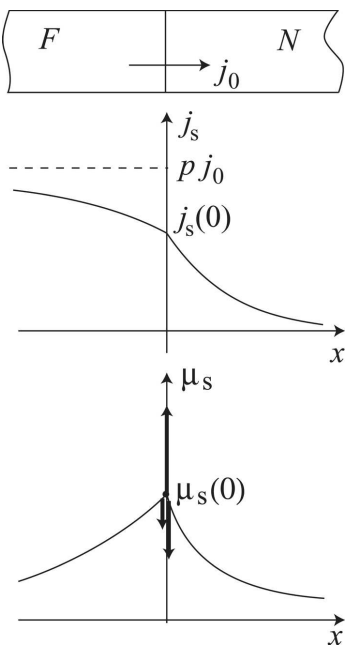

Fig. 2. Dependences $j_{s}(x)$ and $\mu_{s}(x)$ in the $\mathrm{F} / \mathrm{N}$ contact. The arrows in the lower panel demonstrate forces that act on the point $\mu_{s}(0)$ in the mechanical analogy

the spin current is fully absorbed at the $\mathrm{N} / \mathrm{F}$ interface, it seems hardly possible to use an approximation, where the spin potential jumps across the interfaces could be neglected. However, the boundary conditions consistently derived from the microscopic model for the $\mathrm{N} / \mathrm{F}$ contact $[16,17]$ show that the limit of a transparent interface does exist in this case as well. In this limit, the potentials $\mu_{0}$ and $\boldsymbol{\mu}_{s}$ are continuous. The situation is analogous to the case of electrical charge transfer. The difference consists in that it is the spin current $\mathbf{j}_{s}$ that becomes discontinuous at the interfaces. This result has no analog for electrical circuits, where the discontinuity of an electric current is prohibited by the charge conservation law. In the spin case, a certain analog of the current continuity condition exists for interfaces that can be modeled with a static spin-dependent potential. If the interface does not have an associated magnetic moment, i.e. an additional magnetic degree of freedom, then the component of the spin current $\mathbf{j}_{s} \cdot \mathbf{m}$, which is parallel to the magnetization vector, has no jump. Such interfaces are called "spin-inactive". It should be noted that the interfaces can be spin-inactive only for the parallel component of $\mathbf{j}_{s}$. The $\mathrm{N} / \mathrm{F}$ interfaces with a strong ferromagnet are always spin-active for the perpendicular spin-current component.

In works [9-11], it was shown that two continuity conditions

$\left.\boldsymbol{\mu}_{s}\right|_{\mathrm{N}}=\left.\boldsymbol{\mu}_{s}\right|_{\mathrm{F}}$, $\left.\left(\mathbf{j}_{s} \cdot \mathbf{m}\right)\right|_{\mathrm{N}}=\left.\left(\mathbf{j}_{s} \cdot \mathbf{m}\right)\right|_{\mathrm{F}}$

are enough to completely determine the currents in all layers. In this case, no boundary conditions are imposed on the perpendicular components of the spin current, and they can be found automatically from the bulk equations.

\section{Mechanical Analogy}

\section{1. $F / N$ contact}

In order to introduce necessary concepts into consideration, let us at first consider the well-known case of a single $\mathrm{F} / \mathrm{N}$ contact with a spin-inactive interface located at the point $x=0$ between two semiinfinite $\mathrm{F}$ and $\mathrm{N}$ layers [14]. The spin accumulation is determined by Eqs. (4) and (5)/ref), the value of $j_{0}$, and the potentials $\boldsymbol{\mu}_{s}$ at the left and right sides of the interface. As was discussed in Introduction, the spin accumulation occurs only near the interface. At large distances from the interface, the spin accumulation tends to zero, so that $\boldsymbol{\mu}_{s}( \pm \infty)=0$. The value of $\boldsymbol{\mu}_{s}(0)$ has to be found from the continuity conditions for the parallel component of the spin current across the interface.

At the $\mathrm{F} / \mathrm{N}$ contact, both the spin accumulation and the spin current are parallel to the F-layer magnetization at every point: $\boldsymbol{\mu}_{s}(x)=\mu_{s}(x) \mathbf{m}$ and $\mathbf{j}_{s}=j_{s}(x) \mathbf{m}$. The solutions of Eq. (5) look like

$\mu_{s}(x)=\mu_{s}(0) \exp \left(\frac{x}{l_{\mathrm{F}}}\right) \quad(x<0)$,
$\mu_{s}(x)=\mu_{s}(0) \exp \left(-\frac{x}{l_{\mathrm{N}}}\right) \quad(x>0)$,

where $l=\sqrt{D \tau /(2 \rho)}$ is the spin diffusion distance for the given material. The boundary condition (7) takes the form

$p j_{0}-G_{\mathrm{F}} \mu_{s}(0)-G_{\mathrm{N}} \mu_{s}(0)=0$,

where the definition $G=D /(2 l)$ is used for every layer.

The plots of the dependences $\mu_{s}(x)$ and $j_{s}(x)$ are shown in Fig. 2. Their shapes can be understood on the basis of the following mechanical analogy. Let us suppose that the curves $\mu_{s}(x)$ are elastic cords, and the external force $p j_{0}$ is applied upward to their connection point $\mu_{s}(0)$. The elasticity of the cords causes the appearance of the counteracting forces $G_{\mathrm{F}} \mu_{s}(0)$

ISSN 2071-0194. Ukr. J. Phys. 2020. Vol. 65, No. 10 
and $G_{\mathrm{N}} \mu_{s}(0)$, which are proportional to the connection point deviation from the equilibrium position $\mu_{s}(0)=0$. Then Eq. (8) can be regarded as a balance condition for the external force and the elastic reaction forces. In the mechanical analogy, the quantities $G$ play the role of cord stiffness. They contain information about the spin system properties. For example, they are proportional to $1 / l$, so that the reduction of $l$, i.e. the growth of spin relaxation, diminishes the deviation of the connecting point from the equilibrium, i.e. decreases the spin accumulation.

Note that, in the case of $\mathrm{N} / \mathrm{F}$ contact (the particle flux is directed from $\mathrm{N}$ to $\mathrm{F}$ ), Eq. (7) takes the form

$$
-p j_{0}-G_{\mathrm{F}} \mu_{s}(0)-G_{\mathrm{N}} \mu_{s}(0)=0 .
$$

According to the mechanical analogy, the external force drags the point $\mu_{s}(0)$ downward.

By applying the rules of the mechanical analogy described above to the $\mathrm{F} / \mathrm{N}$ and $\mathrm{N} / \mathrm{F}$ contacts, we can easily understand the results obtained for the collinear structures $\mathrm{F} / \mathrm{N} / \mathrm{F}$ and $\mathrm{N} / \mathrm{F} / \mathrm{N}$. In those structures, external forces at the interface drag the connecting points of elastic cords in opposite directions (Fig. 3).

\subsection{Noncollinear structure}

Now, let us consider one of the simplest structures with the alternating-sign function $g(\theta)$, namely, the structure $\mathrm{N} / \mathrm{F}_{1} / \mathrm{N} / \mathrm{F}_{2} / \mathrm{N}$ with $l \rightarrow 0$ in the normal external electrodes [9]. As follows from the results of the previous section, the latter condition provides the equality $\boldsymbol{\mu}_{s}=0$ at the boundaries $\mathrm{N} / \mathrm{F}_{1}$ and $\mathrm{F}_{2} / \mathrm{N}$. The values $\boldsymbol{\mu}_{s}\left(x_{1}\right)=\boldsymbol{\mu}_{1}$ and $\boldsymbol{\mu}_{s}\left(x_{2}\right)=\boldsymbol{\mu}_{2}$ at the interfaces $\mathrm{F}_{1} / \mathrm{N}\left(x=x_{1}\right)$ and $\mathrm{N} / \mathrm{F}_{2}\left(x=x_{2}\right)$ are determined from conditions (7). The solutions of the stationary equation (5) within each of the $\mathrm{F}$ and $\mathrm{N}$ layers have the form

$\boldsymbol{\mu}_{s}(x)=\mathbf{A} \exp \left(-\frac{x}{l}\right)+\mathbf{B} \exp \left(\frac{x}{l}\right)$,

where the constants $\mathbf{A}$ and $\mathbf{B}$ are determined by the vectors $\boldsymbol{\mu}_{s}(L)$ and $\boldsymbol{\mu}_{s}(R)$ at the left and right, respectively, boundaries of the layer. As a result, the vector $\boldsymbol{\mu}_{s}(x)$ in each layer lies in the plane determined by the corresponding vectors $\boldsymbol{\mu}_{s}(L)$ and $\boldsymbol{\mu}_{s}(R)$. The relevant calculations were carried out in works $[9,11]$ (see Appendix 4). Here, let us proceed to the interpretation of the results obtained in the cited works
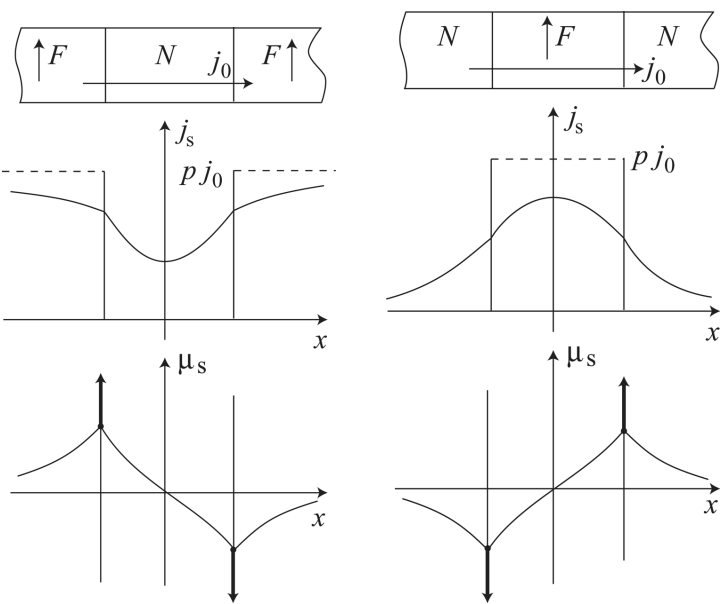

Fig. 3. Dependences $j_{s}(x)$ and $\mu_{s}(x)$ in the collinear structures $\mathrm{F} / \mathrm{N} / \mathrm{F}$ and $\mathrm{N} / \mathrm{F} / \mathrm{N}$. The lower panels demonstrate that, in the mechanical analogy, the directions of the forces acting on the cord-connecting points depend on the arrangement order of the $\mathrm{N}$ and $\mathrm{F}$ layers along the $j_{0}$-current flow

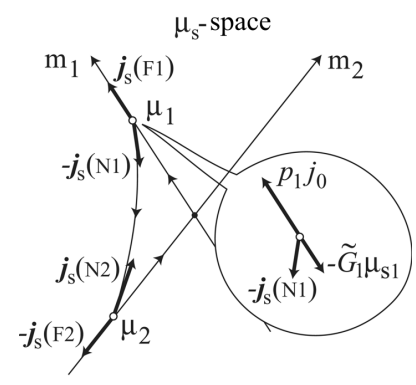

$\boldsymbol{j}_{\mathrm{S}}$-space

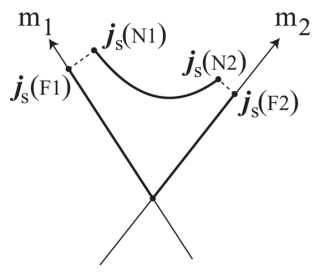

Fig. 4. Hodographs of $\boldsymbol{\mu}_{s}(x)$ and $\mathbf{j}_{s}(x)$

in the mechanical analogy framework. In principle, the vector quantities $\boldsymbol{\mu}_{s}(x)$ could be illustrated in a three-dimensional figure. But since $\boldsymbol{\mu}_{s}(x)$ in each layer varies in a fixed plane, it is enough to consider a two-dimensional hodograph of the motion of the point $\boldsymbol{\mu}_{s}$. In the case of a structure with two ferromagnets, the entire hodograph lies in the plane of the vectors $\mathbf{m}_{1}$ and $\mathbf{m}_{2}$ (Fig. 4, upper panel). In the ferromagnetic layers, the point $\boldsymbol{\mu}_{s}(x)$ moves along the vectors $\mathbf{m}_{1}$ and $\mathbf{m}_{2}$. In the layer of a normal metal, the 
trajectory $\boldsymbol{\mu}_{s}(x)$ connects the points $\boldsymbol{\mu}_{1}$ and $\boldsymbol{\mu}_{2}$. The boundary conditions $(7)$ at the points $x_{1}$ and $x_{2}$ read

$$
\begin{aligned}
& {\left[\mathbf{j}_{s}\left(\mathrm{~F}_{1}\right)-\mathbf{j}_{s}\left(\mathrm{~N}_{1}\right)\right] \cdot \mathbf{m}_{1}=0,} \\
& {\left[\mathbf{j}_{s}\left(\mathrm{~N}_{2}\right)-\mathbf{j}_{s}\left(\mathrm{~F}_{2}\right)\right] \cdot \mathbf{m}_{2}=0,}
\end{aligned}
$$

where the arguments $\mathrm{F}_{1}, \mathrm{~N}_{1}, \mathrm{~N}_{2}$, and $\mathrm{F}_{2}$ denote the points $x_{1}-0, x_{1}+0, x_{2}-0$, and $x_{2}+0$, respectively.

Now, let us proceed to the description of a mechanical analogy in the noncollinear case. In this case, the role of elastic cords is played by the hodograph lines in each layer. The cords in the normal metal and two ferromagnets are connected at two moving points, $\boldsymbol{\mu}_{1}$ and $\boldsymbol{\mu}_{2}$, which can slide along the straight lines $\mathbf{m}_{1}$ and $\mathbf{m}_{2}$ without friction. Let us imagine those points to be small rings encircling the $\mathbf{m}_{1}$ and $\mathbf{m}_{2}$ axes. The axes should be imagined as spaced apart a short distance from each other, so that the rings could pass freely through their "intersection" point. As one can see from Fig. 4, the cords in the ferromagnetic layers form straight lines between the coordinate origin and the points $\boldsymbol{\mu}_{1,2}$, whereas the cord in a normal metal is drawn with a certain sag between the points $\boldsymbol{\mu}_{1}$ and $\boldsymbol{\mu}_{2}$. In the mechanical analogy, the spin currents $\mathbf{j}_{s}$ play the role of the forces with which the cords act on the connecting rings. Indeed, according to formula (3), the spin current in the normal interval is directed tangentially to the hodograph, just like the tension of the elastic cord. The cord departing from the ring as the $x$-coordinate grows acts on the ring with the force $-\mathbf{j}_{s}$, whereas the cord approaching the ring as the $x$-coordinate grows acts on the ring with the force $+\mathbf{j}_{s}$. Since the motion of the rings is confined to the straight lines, only the force components in the directions $\mathbf{m}_{1}$ and $\mathbf{m}_{2}$ (Fig. 4, upper panel) should balance in equilibrium, which brings about conditions (10).

When considering the spin current in the ferromagnets in more detail, one can see that it consists of two terms, e.g., $j_{s}\left(F_{1}\right)=p_{1} j_{0}-\tilde{G}_{1} \mu_{1}$ (the quantity $\tilde{G}_{1}$ is defined in Appendix I). Analogously to the collinear case, the term proportional to the electric current generates an external force that pulls the ring away from the equilibrium position. The second term describes an elastic reaction force that returns the ring back to the equilibrium position; in other words, it diminishes the spin accumulation (Fig. 4, the inset in the upper panel). As one can see, in the case of mechanical analogy, only the forces generated by the electric current in the system can disturb the system from the equilibrium state, which completely corresponds to the physical scenario of the process.

The analogy with the balance of forces can also be extended on Eq. (5) describing the cord shape in the normal metal. Being rewritten in the form

$-\frac{d \mathbf{j}_{s}}{d x}-\frac{\rho}{\tau} \boldsymbol{\mu}_{s}=0$

it is interpreted as the balance between the cord tension forces and the distributed gravitational force applied to each of its infinitesimal elements and directed toward the coordinate origin. It is this gravitational force that results in the "sag" of the cord in a normal metal, which can be observed in Fig. 4. Since it is inversely proportional to $\tau$, the hodograph of $\boldsymbol{\mu}_{s}(x)$ becomes a straight line extending from the point $\boldsymbol{\mu}_{1}$ to the point $\boldsymbol{\mu}_{2}$ in the absence of a spin relaxation in the normal layer $(\tau \rightarrow \infty)$.

Note also that the stationary transport equation (5) and the boundary conditions (6) and (7) can be obtained by finding the extremum of the functional

$S=\sum_{\alpha} \int\left[\frac{1}{D_{\alpha}}\left(p_{\alpha} j_{0}-D_{\alpha} \frac{\nabla_{x} \boldsymbol{\mu}_{s}}{2}\right)^{2}+\frac{\rho_{\alpha} \boldsymbol{\mu}_{s}^{2}}{\tau_{\alpha}}\right] d x$

on the class of continuous functions $\boldsymbol{\mu}_{s}(x)$ with the restriction $\boldsymbol{\mu}_{s}(x) \| \mathbf{m}$ in the ferromagnets. In Eq. (11), the summation is carried out over all layers (subscript $\alpha$ ), and the integration is performed along each layer. The parameters $p_{\alpha}, D_{\alpha}, \tau_{\alpha}$, and $\rho_{\alpha}$ are constant within each layer and depend on its material. From the viewpoint of diffusion transport, this result can be considered as a variant of the principle of minimum entropy production in a weakly nonequilibrium system. From the viewpoint of mechanical analogy, the first term in the integrand corresponds to the elastic energy of the cord, and the second one to the energy associated with the distributed gravitational force.

In general, the mechanical analogy allows the behavior of $\mathrm{F} / \mathrm{N} / \mathrm{F}$ structures to be interpreted at a qualitative level and the results of analytic calculations to be predicted. Let us demonstrate this statement by the example of the properties of spin-transfer torques.

\subsection{Spin-transfer torques and alternating-sign efficiency function}

The spin-transfer torque $\mathbf{T}$ is determined by the spincurrent jump $\Delta \mathbf{j}_{s}$ at the interface between the ferro-

ISSN 2071-0194. Ukr. J. Phys. 2020. Vol. 65, No. 10 
magnetic and normal layers as $\mathbf{T}=(\hbar / 2) \Delta \mathbf{j}_{s}[4]$. According to the boundary conditions $(7)$ and formulas (1), the jumps $\Delta \mathbf{j}_{s}$ and the torques $\mathbf{T}$ are perpendicular to the ferromagnet magnetization. In the structure $\mathrm{N} / \mathrm{F}_{1} / \mathrm{N} / \mathrm{F}_{2} / \mathrm{N}$, the jumps occur only at the interfaces $\mathrm{F}_{1} / \mathrm{N}$ and $\mathrm{N} / \mathrm{F}_{2}$. Then we obtain

$$
\begin{aligned}
\Delta \mathbf{j}_{s 1} & =\mathbf{j}_{s}\left(F_{1}\right)-\mathbf{j}_{s}\left(N_{1}\right)=j_{s}\left(F_{1}\right) \mathbf{m}_{1}-\mathbf{j}_{s}\left(N_{1}\right), \\
\Delta \mathbf{j}_{s 2} & =\mathbf{j}_{s}\left(N_{2}\right)-\mathbf{j}_{s}\left(F_{2}\right)=\mathbf{j}_{s}\left(N_{2}\right)-j_{s}\left(F_{2}\right) \mathbf{m}_{2} .
\end{aligned}
$$

Due to the discontinuities of the spin current, the hodograph of the vector $\mathbf{j}_{s}(x)$ consists of separate segments (Fig. 4, lower panel). The segment corresponding to the normal layer is a curve connecting the points $\mathbf{j}_{s}\left(N_{1}\right)$ and $\mathbf{j}_{s}\left(N_{2}\right)$ with a sag directed toward the coordinate origin. The greater the relaxation, the larger the sags of the hodographs of $\boldsymbol{\mu}_{s}(x)$ and $\mathbf{j}_{s}(x)$, and, simultaneously, the smaller the spin current jumps at the interfaces. In the opposite limit, when the spin relaxation decreases to zero, the hodograph of $\boldsymbol{\mu}_{s}(x)$ approaches a straight line, and the hodograph of $\mathbf{j}_{s}(x)$ transforms into a point, i.e., the spin current in the layer becomes constant.

The directions of the $\Delta \mathbf{j}_{s}$-jumps in the hodograph of $\mathbf{j}_{s}(x)$ depend on the location of the vectors $\mathbf{j}_{s}\left(N_{1}\right)$ and $\mathbf{j}_{s}\left(N_{2}\right)$ relative to the $\mathbf{m}_{1,2}$ lines. The position of those vectors in Fig. 4 corresponds to the positive values of $g_{1}$ and $g_{2}$.

Now let us consider a situation with substantially different properties of the ferromagnets $F_{1}$ and $F_{2}$ (Fig. 5). From the viewpoint of mechanical analogy, the asymmetry means that the rings connecting the elastic cords, $\boldsymbol{\mu}_{1}$ and $\boldsymbol{\mu}_{2}$, are dragged away from the coordinate origin by different forces, $p_{1} j_{0} \mathbf{m}_{1}$ and $-p_{2} j_{0} \mathbf{m}_{2}$. The reaction forces $-\tilde{G}_{1} \boldsymbol{\mu}_{1}$ and $-\tilde{G}_{2} \boldsymbol{\mu}_{2}$ are also different (see Appendix 4). An example of such a situation is shown in Fig. 5 (upper panel). Here, the force $-p_{2} j_{0} \mathbf{m}_{2}$ applied to the ring $\boldsymbol{\mu}_{2}$ was so large that, being transmited through the normal metal cord, it had dragged the ring $\boldsymbol{\mu}_{1}$ to the negative side of the $\mathbf{m}_{1}$ axis. At the same time, the orientation of the tangent line to the N-hodograph at the point $\boldsymbol{\mu}_{2}$ changed: now it passes below the line $\mathbf{m}_{2}$. The corresponding hodograph of $\mathbf{j}_{s}(x)$ is shown in Fig. 5 (lower panel). One can see that the direction of $\Delta \mathbf{j}_{s 2}$ changed, and now the magnetization $\mathbf{m}_{2}$ is repelled from $\mathbf{m}_{1}$. In other words, $g_{2}<0$. Note that, at the moment when $\boldsymbol{\mu}_{1}$ becomes equal to zero, the relative arrangement of $\mathbf{j}_{s}\left(N_{1}\right)$ and $\mathbf{m}_{1}$ and, hence, the direc-

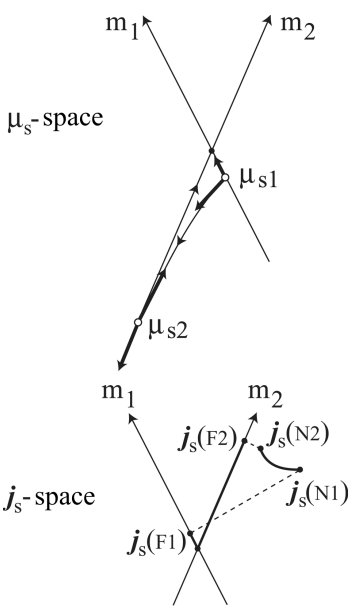

Fig. 5. Hodographs of $\boldsymbol{\mu}_{s}(x)$ and $\mathbf{j}_{s}(x)$ in the case $g_{2}<0$

tion of $\Delta \mathbf{j}_{s 1}$ do not change, i.e., the sign of $g_{2}$ remains positive.

In our example, the "drag" of the ring $\boldsymbol{\mu}_{1}$ takes place owing to the growth of the force $-p_{2} j_{0} \mathbf{m}_{2}$. On the other hand, it can also occur due to a reduction of the counteracting force $-\tilde{G}_{2} \boldsymbol{\mu}_{2}$. In this case, the ring $\boldsymbol{\mu}_{2}$ would also further away from the equilibrium position and pull the ring $\boldsymbol{\mu}_{1}$ behind itself by means of the normal metal cord.

\section{Conclusions}

The mechanical analogy allows the behavior of the diffusion system to be predicted on the basis of qualitative considerations. For example, it becomes evident that if the angle $\theta$ between the magnetizations $\mathbf{m}_{1}$ and $\mathbf{m}_{2}$ exceeds $90^{\circ}$, no force directed along $-\mathbf{m}_{2}$ would be capable of dragging the point $\boldsymbol{\mu}_{1}$ through zero, because the component of such a force on $\mathbf{m}_{1}$ is positive (here, we assume $j_{0}>0$ ). Accordingly, the change of the $g_{2}$-sign is possible, only if $0 \leqslant \theta<\pi / 2$, in accord with the formulas obtained in works $[9,11]$ (see Appendix II). The mechanical analogy makes it also evident that, in the case $0 \leqslant \theta<\pi / 2$, a sufficiently large polarization $p_{1}$ will change the sign of $g_{1}$ without changing the sign of $g_{2}$.

Finally, it becomes obvious that two efficiency functions cannot change their signs simultaneously. Indeed, for the sign of $g$ to change, it is necessary to drag the corresponding ring through the point, where the axes intersect. In the mechanical analogy, the both external forces act on the rings in the directions away from the equilibrium positions. Therefore, 
if the asymmetry increases, at least one of the rings will shift in the direction of the applied force, but not opposite to it ${ }^{2}$.

In diffusive structures consisting of more than two ferromagnetic elements, the behavior of the spin accumulation is represented by a planar hodograph in each normal-metal layer and by a linear hodograph along the magnetization axis in each ferromagnetic layer. In structures with noncoplanar magnetizations, there may be situations in which the planes of hodographs of normal layers are different. But this complication does not prevent all hodographs to be simultaneously presented in two-dimensional diagrams like Figs. 4 and 5. The fact is that the planes of the hodographs of two normal layers separated by a ferromagnetic gap always possess a common line; it is the axis of ferromagnet magnetization. Therefore, a three-dimensional hodograph of a multilayer structure can always be "folded" into a flat one.

The mechanical analogy continues to be valid for multilayer structures, because the balance of imaginary forces applied to the connecting points between the elastic cords will be preserved at each interface between the layers. Of course, as the number of elements in the system increases, a possibility to intuitively find the shape of a balanced mechanical configuration for the cords decreases. Nevertheless, even in this case, the graphical interpretation of the analytic or numerical solutions in the hodograph form remains to be a useful way of visualization, and the mechanical analogy can make it easy to understand the response of a system to small variations of its parameters.

The work was supported in the framework of the NSF grant DMR-0847159.

\section{APPENDIX I \\ Distribution of Spin Accumulation and Spin Current}

In this appendix, the results of calculations for the $\mathrm{N} / \mathrm{F}_{1} / \mathrm{N} / \mathrm{F}_{2} / \mathrm{N}$ structure are presented. The thicknesses of the ferromagnetic layers $\mathrm{F}_{1}$ and $\mathrm{F}_{2}$ equal $d_{1}$ and $d_{2}$, respectively,

\footnotetext{
2 One may ask what will happen in the case of negative spin polarization, $p<0$ or $D_{\uparrow}<D_{\downarrow}$, in either or both layers. Unfortunately, it will only result in the overdetermination of the direction of the corresponding vector $\mathbf{m}$ to the opposite one.
}

and the thickness of the normal gap between them is $d_{\mathrm{N}}$. The spin diffusion distances are $l_{1}$ and $l_{2}$ in the ferromagnets and $l_{\mathrm{N}}$ in the normal layer. In the external normal electrodes, the spin diffusion distance is assumed to tend to zero, so that the spin accumulation equals zero at the interfaces with them. At the $\mathrm{F}_{1} / \mathrm{N}\left(\right.$ at $\left.x=x_{1}\right)$ and $\mathrm{N} / \mathrm{F}_{2}\left(\right.$ at $\left.x=x_{2}\right)$ interfaces, the spin accumulation is denoted as $\boldsymbol{\mu}_{1}$ and $\boldsymbol{\mu}_{2}$, respectively. The function $\boldsymbol{\mu}(x)$ is given by the following formulas: in $\mathrm{F}_{1}$ (at $\left.x_{1}-d_{1}<x<x_{1}\right)$,

$\boldsymbol{\mu}(x)=\frac{\boldsymbol{\mu}_{1} \sinh \frac{x-\left(x_{1}-d_{1}\right)}{l_{1}}}{\sinh \frac{d_{1}}{l_{1}}} ;$

in $\mathrm{N}\left(\right.$ at $\left.x_{1}<x<x_{2}\right)$,

$\boldsymbol{\mu}(x)=\frac{\boldsymbol{\mu}_{1} \sinh \frac{x_{2}-x}{l_{\mathrm{N}}}+\boldsymbol{\mu}_{2} \sinh \frac{x-x_{1}}{l_{\mathrm{N}}}}{\sinh \frac{d_{\mathrm{N}}}{l_{\mathrm{N}}}} ;$

and in $\mathrm{F}_{2}\left(\right.$ at $\left.x_{2}<x<x_{2}+d_{2}\right)$,

$\boldsymbol{\mu}(x)=\frac{\boldsymbol{\mu}_{2} \sinh \frac{\left(x_{2}+d_{2}\right)-x}{l_{2}}}{\sinh \frac{d_{2}}{l_{2}}}$

Taking the definition $G=D /(2 l)$ for each layer into account, the following expressions for the spin currents are obtained: in $\mathrm{F}_{1}\left(\right.$ at $\left.x_{1}-d_{1}<x<x_{1}\right)$,

$\mathbf{j}_{s}(x)=p_{1} j_{0}-G_{1} \frac{\boldsymbol{\mu}_{1} \cosh \frac{x-\left(x_{1}-d_{1}\right)}{l_{1}}}{\sinh \frac{d_{1}}{l_{1}}}$,

in $\mathrm{N}\left(\right.$ at $\left.x_{1}<x<x_{2}\right)$,

$\mathbf{j}_{s}(x)=G_{\mathrm{N}} \frac{\boldsymbol{\mu}_{1} \cosh \frac{x_{2}-x}{l_{\mathrm{N}}}-\boldsymbol{\mu}_{2} \cosh \frac{x-x_{1}}{l_{\mathrm{N}}}}{\sinh \frac{d_{\mathrm{N}}}{l_{\mathrm{N}}}}$,

and in $\mathrm{F}_{2}\left(\right.$ at $\left.x_{2}<x<x_{2}+d_{2}\right)$,

$\mathbf{j}_{s}(x)=p_{2} j_{0}+G_{2} \frac{\boldsymbol{\mu}_{2} \cosh \frac{\left(x_{2}+d_{2}\right)-x}{l_{2}}}{\sinh \frac{d_{2}}{l_{2}}}$.

At the $\mathrm{F}_{1} / \mathrm{N}$ and $\mathrm{N} / \mathrm{F}_{2}$ interfaces,

$\mathbf{j}_{s}\left(F_{1}\right)=p_{1} j_{0}-\tilde{G}_{1}\left(d_{1}\right) \boldsymbol{\mu}_{1}$,

$\mathbf{j}_{s}\left(N_{1}\right)=\tilde{G}_{\mathrm{N}}\left(d_{\mathrm{N}}\right)\left(\boldsymbol{\mu}_{1}-\frac{1}{\operatorname{ch} d_{\mathrm{N}} / l_{\mathrm{N}}} \boldsymbol{\mu}_{2}\right)$,

$\mathbf{j}_{s}\left(N_{2}\right)=\tilde{G}_{\mathrm{N}}\left(d_{\mathrm{N}}\right)\left(\frac{1}{\operatorname{ch} d_{\mathrm{N}} / l_{\mathrm{N}}} \boldsymbol{\mu}_{1}-\boldsymbol{\mu}_{2}\right)$,

$\mathbf{j}_{s}\left(F_{1}\right)=p_{2} j_{0}+\tilde{G}_{2}\left(d_{2}\right) \boldsymbol{\mu}_{2}$.

where the notation $\tilde{G}(d)=G \operatorname{coth}(d / l)$ was used.

Using expressions (13) and boundary conditions (7), the following formulas are obtained for the spin accumulation at the interfaces:

$\left(\begin{array}{l}\mu_{1} \\ \mu_{2}\end{array}\right)=\frac{j_{0}}{\operatorname{Det}}\left|\begin{array}{cc}A_{2} & -B \cos \theta \\ B \cos \theta & -A_{1}\end{array}\right|\left(\begin{array}{l}p_{1} \\ p_{2}\end{array}\right)$,

where

$A_{1}=\tilde{G}_{1}\left(d_{1}\right)+\tilde{G}_{\mathrm{N}}\left(d_{\mathrm{N}}\right)>0$,

$A_{2}=\tilde{G}_{2}\left(d_{2}\right)+\tilde{G}_{\mathrm{N}}\left(d_{\mathrm{N}}\right)>0$,

$B=\frac{\tilde{G}_{\mathrm{N}}\left(d_{\mathrm{N}}\right)}{\cosh \frac{d_{\mathrm{N}}}{l_{\mathrm{N}}}}>0$, Det $=A_{1} A_{2}-B^{2} \cos ^{2} \theta$.

ISSN 2071-0194. Ukr. J. Phys. 2020. Vol. 65, No. 10 


\section{APPENDIX II}

\section{Spin-Transfer Torques}

The spin current jumps are determined by formulas (12). Substituting relations (13) into them, the following expressions are obtained:

$\Delta \mathbf{j}_{s 1}=\frac{G_{\mathrm{N}}\left(d_{\mathrm{N}}\right)}{\cosh \frac{d_{\mathrm{N}}}{l_{\mathrm{N}}}} \mu_{2}\left[\mathbf{m}_{1} \times\left[\mathbf{m}_{2} \times \mathbf{m}_{1}\right]\right]$,
$\Delta \mathbf{j}_{s 2}=\frac{G_{\mathrm{N}}\left(d_{\mathrm{N}}\right)}{\cosh \frac{d_{\mathrm{N}}}{l_{\mathrm{N}}}} \mu_{1}\left[\mathbf{m}_{2} \times\left[\mathbf{m}_{1} \times \mathbf{m}_{2}\right]\right]$.

All we need to do is to substitute $\mu_{1}$ and $\mu_{2}$ from formulas (14)

To analyze the behavior of the efficiency functions, at first, we should prove the inequalities $0<B<A_{1,2}$. Using them, we can show that the signs of $g_{1,2}$ change from positive to negative, if the condition $\cos \theta>0$ and either of the inequalities

$$
\begin{aligned}
& \frac{p_{2}}{p_{1}}>\frac{A_{2}}{B \cos \theta}>1 \quad \Rightarrow \quad g_{2}<0, \\
& \frac{p_{2}}{p_{1}}<\frac{B \cos \theta}{A_{1}}<1 \quad \Rightarrow \quad g_{1}<0 .
\end{aligned}
$$

are satisfied simultaneously. Conditions (15) demonstrate that two efficiency functions cannot change their signs simultaneously. For one of them to change its sign, it is necessary that a certain threshold of the structural asymmetry has to be exceeded.

1. L. Berger. Low-field magnetoresistance and domain drag in ferromagnets. J. Appl. Phys. 49, 2156 (1978).

2. L. Berger. Possible existence of a Josephson effect in ferromagnets. Phys. Rev. B 33, 1572 (1986).

3. L. Berger. Emission of spin waves by a magnetic multilayer traversed by a current. Phys. Rev. B 54, 9353 (1996).

4. J. C. Slonczewski. Current-driven excitation of magnetic multilayers. J. Magn. Magn. Mater. 159, L1 (1996).

5. V.K. Dugaev, V.R. Vieira, P.D. Sacramento, J. Barnas, M.A.N. Araujo, J. Berakdar. Current-induced motion of a domain wall in a magnetic nanowire. Phys. Rev. B 74, 054403 (2006)

6. A.M. Pogorilyi, S.M. Ryabchenko, O.I. Tovstolytkin. Spintronics. Main phenomena. Development trends. Ukr. Fiz. Zh. Ogl. 6, 37 (2010) (in Ukrainian).

7. J.C. Slonczewski. Currents and torques in metallic magnetic multilayers. J. Magn. Magn. Mater. 247, 324 (2002).

8. M.D. Stiles, A. Zangwill, Anatomy of spin transfer. Phys. Rev. B 66, 014407 (2002).

9. A.A. Kovalev, A. Brataas, G.E.W. Bauer. Spin-transfer in diffusive ferromagnet-normal metal systems with spin-flip scattering. Phys. Rev. B 66224424 (2002).
10. J. Manschot, A. Brataas, G.E.W. Bauer. Nonmonotonic angular magnetoresistance in asymmetric spin valves. Phys. Rev. B 69092407 (2004).

11. J. Barnas, A. Fert, M. Gmitra, I. Weymann, V.K. Dugaev. From giant magnetoresistance to current-induced switching by spin transfer. Phys. Rev. B 72, 024426 (2005).

12. M. Gmitra, J. Barnas. Current-driven destabilization of both collinear configurations in asymmetric spin valves. Phys. Rev. Lett. 96, 207205 (2006).

13. P. Balaz, M. Gmitra, J. Barnas. Current-pulse-induced magnetic switching in standard and nonstandard spinvalves: Theory and numerical analysis. Phys. Rev. B 79, 144301 (2009).

14. P.C. van Son, H. van Kempen, P. Wyder. Boundary resistance of the ferromagnetic-nonferromagnetic metal interface. Phys. Rev. Lett. 58, 2271 (1987).

15. T. Valet, A. Fert. Theory of the perpendicular magnetoresistance in magnetic multilayers. Phys. Rev. B 48, 7099 (1993).

16. A. Brataas, Yu.V. Nazarov, G.E.W. Bauer. Finite-element theory of transport in ferromagnet-normal metal systems. Phys. Rev. Lett. 84, 2481 (2000).

17. A. Brataas, Yu.V. Nazarov, G.E.W. Bauer. Spin-transport in multi-terminal normal metal-ferromagnet systems with non-collinear magnetizations. Eur. Phys. J. B 22, 99 (2001).

18. C. Heide. Spin currents in magnetic films. Phys. Rev. Lett. 87, 197201 (2001).

19. C. Heide. Effects of spin accumulation in magnetic multilayers. Phys. Rev. B 65, 054401 (2002).

20. E.I. Rashba. Diffusion theory of spin injection through resistive contacts. Eur. Phys. J. B 29513 (2002).

Received 10.07.20

Translated from Ukrainian by O.I. Voitenko

Я.Б. Базалій

ІНТЕРПРЕТАЦІЯ ЗНАКОЗМІННОГО

СПIН-ТРАНСФЕРНОГО МОМЕНТУ В F/N/F

СТРУКТУРАХ ЗА ДОПОМОГОЮ

МЕХАНІЧНОЇ АНАЛОГЇ̈

$\mathrm{P}$ е $з$ ю м е

Обговорюється зручна інтерпретація розподілу спінового накопичення та спінових струмів у магнітних наноструктурах з дифузійним переносом. Запропоновано механічну аналогію, яка дозволяє на якісному рівні зрозуміти міру впливу різних параметрів на величину спін-трансферних моментів і наочно пояснюе знакозмінність спін-трансферного моменту в асиметричних $\mathrm{F} / \mathrm{N} / \mathrm{F}$ структурах. 